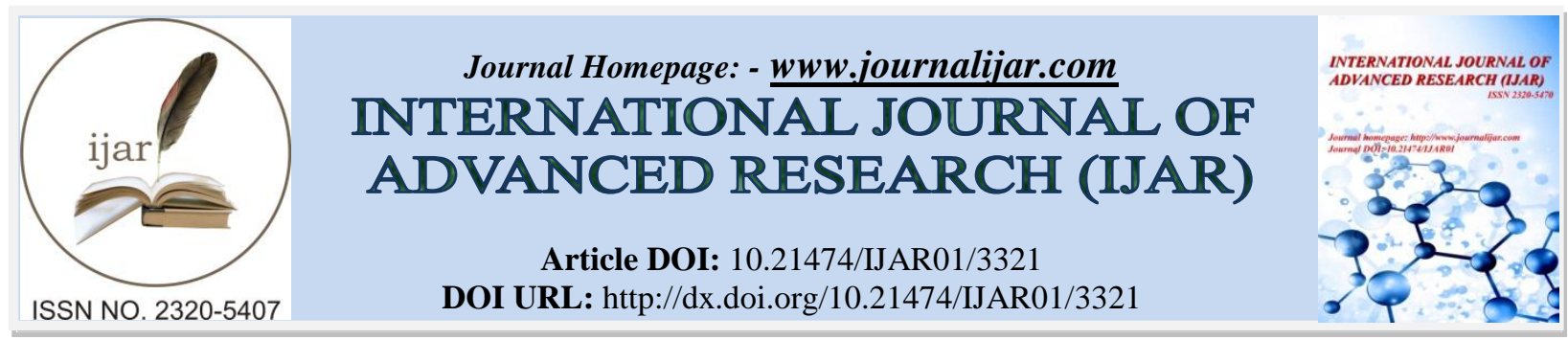

RESEARCH ARTICLE

\title{
CORRELATION OF TIRADS [THYROID IMAGING REPORTING AND DATA SYSTEM] AND HISTOPATHOLOGICAL FINDINGS IN EVALUATION OF THYROID NODULES.
}

Simmi Bhatnagar, Jaswinder Kaur Mohi, Navkiran Kaur, Amarjeet Kaur and Lovepreet Singh.

\section{Manuscript Info}

Manuscript History

Received: 26 December 2016

Final Accepted: 22 January 2017

Published: February 2017

Key words:-

Echogenicity,

Microcalcification, Irregular contours.

\section{Abstract}

Purpose: Ultrasound [US] is an excellent noninvasive modality to evaluate thyroid nodules.Aim of our study was to characterize the thyroid nodules according to grey scale sonographic features into high suspicious ,probably benign, benign aspects and normal thyroid using TIRADS scoring system and to characterize the thyroid nodule according to the sonographic features into a specific TIRADS stage and correlate the results with the histopathological examination findings wherever possible.

Material And Methods: The prospective study was carried out on 60 patients referred to Department of Radiodiagnosis, Rajindra Hospital,Patiala. All the patients were subjected to detailed history taking,clinical examination and routine laboratory investigations.All thyroid nodules were characterized according to the internal component[solid, mixed or cystic], the margins, echogenicity [hyperechoic, Isoechoic,hypoechoic, marked hypoehoic], evidence of calcification [microcalcification if less than $3 \mathrm{~mm}$ and macrocalcification if more than $3 \mathrm{~mm}$ with acoustic shadowing]and the shape[taller than wide if greater in anteroposterior dimension than in its transverse dimension and wider than tall]. Using the Modified Russ Classification, each nodule was classified into TIRADS categories [1,2,3,4A,4B and 5] based on ultrasound features.The patients were referred to Department of Pathology, Government Medical College Patiala and FNAC was done. The US findings were correlated with FNAC and data was analyzed statistically.

Results: Based on various ultrasound characteristics of thyroid nodules,TIRADS score was given to each thyroid nodule and then FNAC was done.The results of histopathology were correlated with ultrasound features and statistical analysis was done calculating sensitivity, specificity,positive predictive value and negative predictive value for each feature.The sensitivity and specificity for Irregular contours were $44.4 \%$ and $94.12 \%$,for taller than wide were $22.22 \%$ and $100 \%$,for microcalcification were $33.3 .3 \%$ and $94.12 \%$,for marked hypoechogenicity was 78 and $70.89 \%$ and for solid consistency were 89 and $70.5 \%$. The risk of malignancy was found to increase from TIRADS3 to TIRADS5 in this study. All the cases [100\%] of TIRADS 5 turned out to be malignant on histopathology.

Conclusion: Radiologists should be aware of usefulness of specific ultrasound features of thyroid nodules like Irregular contours, taller 
than wide configuration, microcalcification, marked hypoechogenicity and solid consistency collectively taken as TIRADS for better differentiation of benign from malignant or potentially malignant lesions that warrant further diagnostic evaluation. The risk of malignancy was found to increase from TIRADS 3 to TIRADS 5 when different TIRADS categories were confronted with results of pathology and risk of malignancy was calculated.

Copy Right, IJAR, 2017,. All rights reserved.

\section{Introduction:-}

Thyroid nodules are a commonly encountered clinical problem in daily practice.With the increased use of high resolution ultrasound during last two decades, the detection rate has gone up[1].This is in parallel to increased incidence of thyroid cancer worldwide since malignancy comprises approximately 5\% of all Thyroid nodules irrespective of size[2]. The initial evaluation of thyroid nodules should always focus on exclusion of malignancy[4].The prevalence of non-palpable nodules has increased recently as a consequence of the increasing application of ultrasound[5].Nowadays, the use of high technology US equipment may detect nodules as small as 2 to $3 \mathrm{~mm}$, which raises the question of which thyroid nodules are clinically relevant for further evaluation[3].Various ultrasound characteristics of thyroid nodules have proven to have predictive diagnostic value in suspicion of malignancy,with particular focus on which nodules should be subjected to US guided fine needle aspiration [FNA].Thyroid US should always include evaluation of neck for abnormal lymph nodes. When abnormal lymph nodes are present, biopsy for cytology and thyroglobulin washout should be performed at the same time as the nodule biopsy [6].Fine Needle Aspiration Cytology [FNAC] is considered the most reliable test for diagnosis of thyroid nodules. FNAC is recommended for palpable nodules, but the indication for this procedure in non-palpable nodules is a matter of controversy. Some clinicians recommend ultrasound guided FNAC while others consider that a clinical follow up [neck palpation] is sufficient in absence of history of familial thyroid cancer or head/neck irradiation[5].Neck US has long been used to evaluate the size,character and location of thyroid nodules, monitor nodule growth and identify loco-regional lymphadenopathy. Many studies have classified specific US characteristics predictive of malignant nodules.For instance, current US characteristics that are strongly correlated with malignancy include intranodular vascularity,the presence of microcalcifications, a taller than wider pattern, hypoechogenicity and spiculated margins[7]. Although controversial, the current guidelines state that if any one of the above findings is observed,with the exception of increased intranodular vascularity, the nodule should be defined as suspicious for malignancy.

\section{Materials And Methods:-}

In this prospective study, we considered 60patients referred to Department of Radiodiagnosis, Rajindra Hospital Patiala. Prior permission regarding this study was taken from hospital authorities. The Thyroid was imaged with high frequency linear array transducer with the patient in supine position with neck extended by placement of a pillow beneath the shoulders.The number, location, size in three dimensions and characteristics of nodules were documented along with examination of neck adenopathy. Ultrasound was performed with Philips Envisor with a $5.5 \mathrm{MHz}$ probe. All the patients were subjected to detailed history taking, clinical examination, routine laboratory investigations and high resolution ultrasonography. All Thyroid nodules were characterized according to the internal components [solid, cystic, mixed], the margins, echogenicity, type of calcification if present [micro less than $3 \mathrm{~mm}$ and macro if more than $3 \mathrm{~mm}$ ], the shape of the nodule [characterised as "taller than wider" [greater in anteroposterior dimension than in its transverse diameter and "wider than taller"]. Using the Modified Russ classification,each nodule was classified into TIRADS category $[1,2,3,4 \mathrm{~A}, 4 \mathrm{~B}, 5]$ based on ultrasound features. The findings were correlated with FNAC.Then data was analyzed statistically.

\section{Tirads Classification Alogrithm:-}

The terminology of TIRADS was first used by Hogarth et al.They described 10 ultrasound patterns of thyroid nodules and related the rate of malignancy according to the pattern. The followingcategories were established:

TIRADS 1:Normal Thyroid gland

TIRADS 2:Benign conditions [0\%malignancy]

TIRADS 3:Probably benign nodules [5\% malignancy] 
TIRADS 4:Suspicious nodules [5-80\%malignancy].A subdivision into $4 \mathrm{a}$ [malignancy between 5and10\%] and $4 \mathrm{~b}$ [ malignancy between 10 and $80 \%$ ] was optional.

TIRADS 5:Probably malignant nodules[malignancy $80 \%$ ]

TIRADS 6:Category included biopsy proven malignant nodules.

\section{Tirads Classification Alogrithm}

\begin{tabular}{|c|c|}
\hline $\begin{array}{ll}\text { HIGH SUSPICIOUS ASPECTS } \\
\text { - Taller than wide shape } \\
\text { - } \quad \text { Irregular or microlobulated margins } \\
\text { - Microcacifications } \\
\text { - } \quad \text { Marked hypoechogenicity }\end{array}$ & $\begin{array}{l}\geq 3 \text { signs and /or adenopathy } \\
\text { TIRADS } 5 \\
1 \text { or } 2 \text { signs and no adenopathy } \\
\text { TIRADS } 4 \mathrm{~B}\end{array}$ \\
\hline $\begin{array}{l}\text { LOW SUSPICIOUS ASPECTS } \\
\text { - None of the high suspiciousaspects } \\
\text { - Moderately hypoechogenic }\end{array}$ & TIRADS 4A \\
\hline $\begin{array}{l}\text { PROBABLY BENIGN ASPECTS } \\
\text { - None of the high suspicious aspects } \\
\text { - Isoechogenicity } \\
\text { - Hyperechogenic }\end{array}$ & TIRADS 3 \\
\hline $\begin{array}{l}\text { BENIGN ASPECTS } \\
\text { - Simple cyst } \\
\text { - Spongiform nodule } \\
\text { - "White knight" aspect } \\
\text { - Isolated microcalcification } \\
\text { - Typical subacute thyroiditis }\end{array}$ & TIRADS 2 \\
\hline $\begin{array}{l}\text { Normal } \\
\text { Thyroid USG }\end{array}$ & TIRADS 1 \\
\hline
\end{tabular}

Thyroid USG

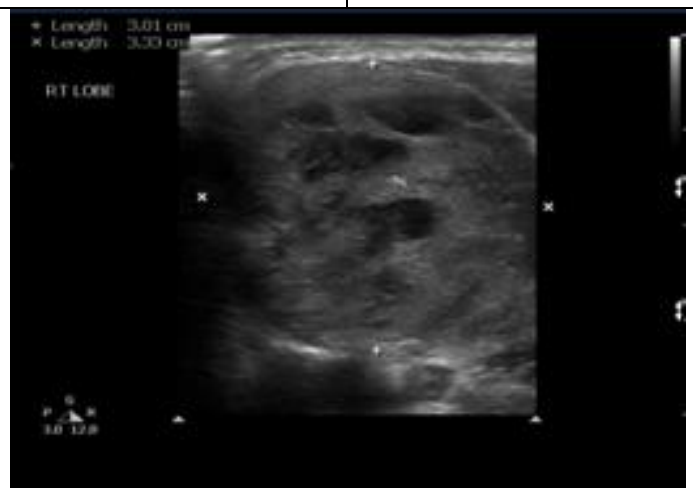

Figure 1:- A well defined wider than tall hyperechoiec nodule seen in the right lobe of thyroid with few cystic areas with no calcification. (TIRADS CATEGORY III)

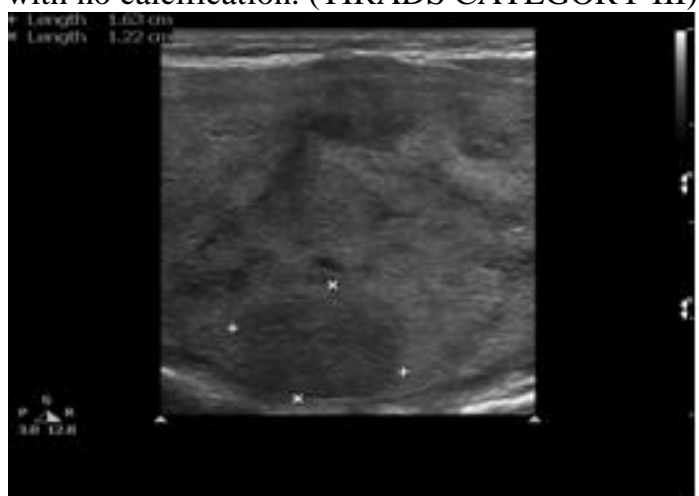

Figure 2:- A well defined moderately hypoechoiec area is seen in the thyroid with no calcification.(TIRADS CATEGORY IV A) 


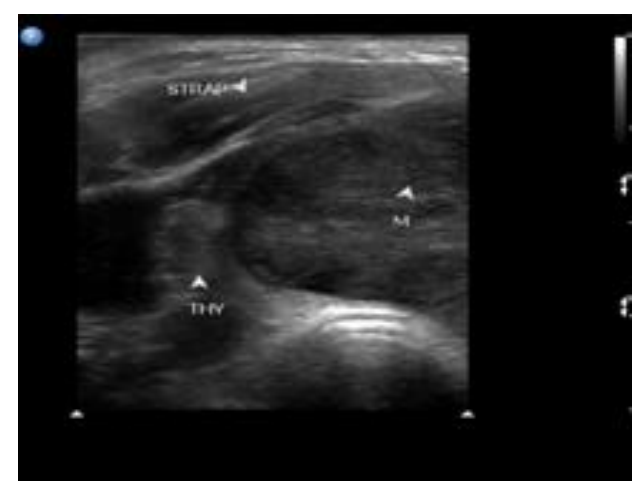

Figure 3:- An ill defined markedly hypoechoiec nodule as compared to strap muscles

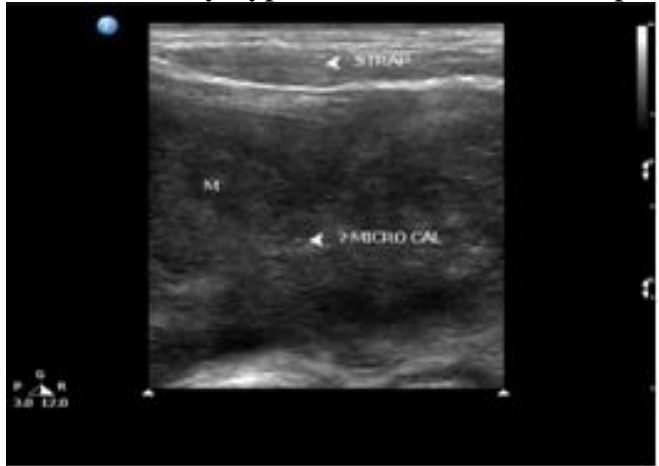

Figure 4:- An ill defined taller than wide markedly hypoechoiec nodule with micro calcifications.(TIRADS CATEGORY V)

\section{Results:-}

Our study consisted of 60 patients with male to female ratio of 1:5.1. $16.67 \%$ were males and $83.33 \%$ were females.Purely solid nodules were seen in 23 [38.3\%] cases. Solid nodules with cystic components were seen in 12 [20\%] cases. Purely cystic nodules were seen in 25 [41.7\%] cases out of 60.Hyperechoic nodules were seen in 14 [23.33\%]cases whereas Isoechoic nodules were seen in 18[30\%] cases.Hypoechoic nodules were seen in 06[10\%] cases and marked hypoechoicnodules were seen in 22 [36.7\%] cases. Sharp well defined margins were seen in53[88.3\%] of cases. Poorly defined margins were seen in 07[11.7\%] of cases.Microcalcification was seen in $06[10 \%]$ cases and macrocalcification was seen in 20[33.3\%] cases. No calcification was seen in 34 [33.3\%]cases. Taller than wide shape of the nodules was seen in 02 [3.33\%] of cases and wider than tall shape was seen in 58[96.7\%]cases.In our study on US, we diagnosed 52 nodulesas benign and 8 nodules as malignant nodules.15 [28.84\%] of the benign nodules were solid and $08[100 \%$ ] of the malignant nodules were solid.12 [23.07\%] of the benign nodules were mixed while none of the malignant nodules was mixed. 25 [48\%] of the benign nodules were cystic and none of the malignant nodules was cystic. On correlation of echogenicity of nodules on ultrasound with histopathological findings, 15 [28.3\%] nodules were markedly hypoechoic and 07 of them turned out to be malignant while 14 [26.4\%]were hyperechoic and none of them turned out to be malignant. On correlation of margins of nodules with histopathological findings 53[94.64\%]cases with well defined margins turned out benign on histopathology and04[100\%] of cases having ill defined margins were found to be malignant. None of the malignant nodules showed well defined margins.On correlation of pattern of calcification and histopathology,microcalcification was seen in 03[13\%]of the benign nodules and 03[100\%] of malignant nodules.Macrocalcification was seen in 20[87\%] of benign nodules. None of the malignant nodules showed macrocalcification.Taller than wide configuration was seen in $02[100 \%$ ] of the malignant nodules and wider than taller configuration was seen in 58[100\%] of the benign nodules. Major ultrasound featuressuggestive of malignancy were analyzed with respect to TIRADS category.Sensitivity, specificity, positive predictive value and negative predictive value were calculated for each feature (Table1). Diagnostic index of individual grey scale sonographic criteria for predicting malignancy in Thyroid nodules was calculated. 
Table 1:- Ultrasound features and their statistical parameters

\begin{tabular}{|l|l|l|l|l|}
\hline Ultrasound feature & True positive & False positive & True negative & False negative \\
\hline $\begin{array}{l}\text { IRREGULAR } \\
\text { CONTOURS }\end{array}$ & O4 & 03 & 48 & O5 \\
\hline TALLER THAN WIDE & 02 & 00 & 51 & 07 \\
\hline MICROCALCIFICATION & 03 & 03 & 48 & 06 \\
\hline $\begin{array}{l}\text { MARKED } \\
\text { HYPOECHOGENICITY }\end{array}$ & 07 & 15 & 36 & 02 \\
\hline SOLID CONSISTENCY & 08 & 15 & 36 & 01 \\
\hline
\end{tabular}

The sensitivity and specificity for Irregular contours were $44.4 \%$ and $94.12 \%$,for taller than wide was $22.22 \%$ and $100 \%$,for microcalcification were $33.3 \%$ and $94.12 \%$, for marked hypoechogenicity were 78 and $70.89 \%$ and for solid consistency were 89 and $70.5 \%$.

Table 2:- Tirads Categories Of Various Nodules

\begin{tabular}{|l|l|}
\hline TIRADS CATEGORY & NO OF NODULES AS PER TIRADS CLASSIFICATION \\
\hline TIRADS 2 & 10 \\
\hline TIRADS 3 & 29 \\
\hline TIRADS 4A & 12 \\
\hline TIRADS 4B & 05 \\
\hline TIRADS 5 & 04 \\
\hline
\end{tabular}

The different TIRADS categories were compared with the results of pathology and risk of malignancy was calculated.The risk of malignancy was found to increase from TIRADS3 to TIRADS 5 (Table 3).

Table3:- Tirads Categories And Risk Of Malignancy

\begin{tabular}{|l|l|l|l|l|}
\hline $\begin{array}{l}\text { TIRADS } \\
\text { CATEGORY }\end{array}$ & PATHOLOGY & & TOTAL & $\begin{array}{l}\text { RISK OF } \\
\text { MALIGNANCY[\%] }\end{array}$ \\
\hline & BENIGN & MALIGNANT & & \\
\hline TIRADS2 & 10 & 00 & 10 & 00 \\
\hline TIRADS 3 & 28 & 01 & 29 & 3.4 \\
\hline TIRADS 4A & 11 & 01 & 12 & 8.3 \\
\hline TIRADS 4B & 02 & 03 & 05 & 60 \\
\hline TIRADS 5 & 00 & 04 & 04 & 100 \\
\hline
\end{tabular}


Table 4:- Bar diagram shows number of nodules according to TIRADS categories and their charaterization into benign and malignant.

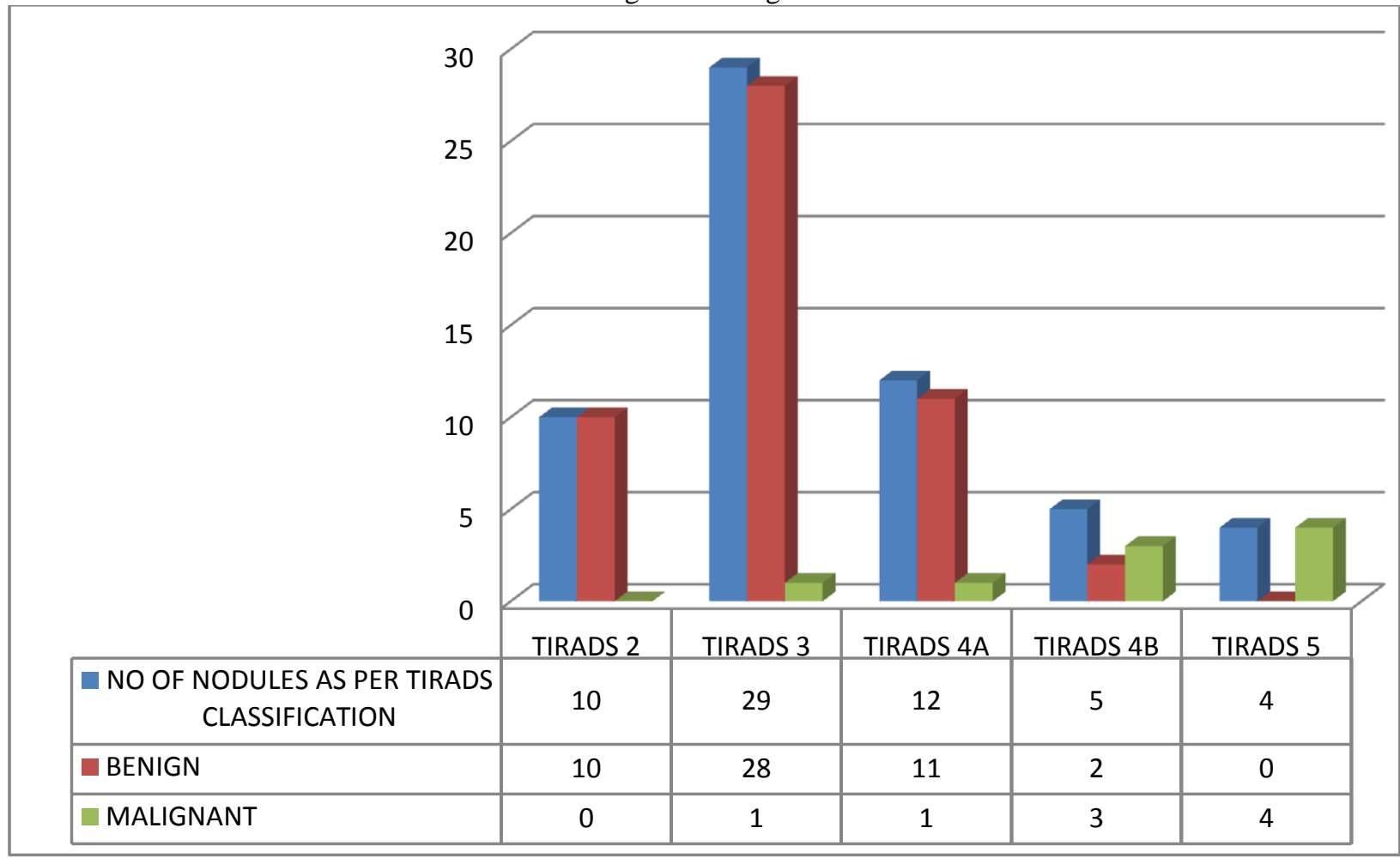

TIRADS categories and diagnostic performance of ultrasound was analysed.Total cases of TIRADS 2,3,4A were 51 out of which 49 were benign and 2 were malignant.Total cases of TIRADS 4B and 5 were 9 out of which 7 were malignant.Out of 51cases, 9 cases were malignant.

\section{Discussion:-}

In present study, 60 cases of nodular thyroid disease were studied by grey scale ultrasonography. We differentiated Thyroid nodules into highly suspicious, low suspicious, probably benign, benign nodules and normal thyroid as per TIRADS scoring system using grey scale scoring sonographic features viz internal components [solid, mixed or cystic], the margins,echogenicity,evidence of calcification and shape. Grey scale ultrasonography was the first investigation to be requested in all cases of thyroid swelling in our study because of it being noninvasive,simple and without radiation exposure.In our study, solid consistency as a predictor for malignancy had sensitivity of $70.5 \%$, positive predictive value of $34.7 \%$ and negative predictive value of $97.3 \%$. Study conducted by Aggarwal et al[11] showed that solid echotexture had sensitivity of $54.5 \%$ and specificity of $64.3 \%$. koike et al [12] got sensitivity of $83.4 \%$ and specificity of $81.8 \%$ of solid echotexture of malignant nodules.In our study hypoechogenicity as criteria for predicting malignancy had sensitivity of $78 \%$,specificity of $70.89 \%$,positive predictive value of $31.82 \%$ and negative predictive value of $94.7 \%$.Koike et al[12] got sensitivity of $95 \%$ and specificity of $51.4 \%$. Raho et al[10] reported that hypoechogenicity had specificity of $48.6 \%$ and sensitivity of $66.6 \%$, positive predictive value of $34.4 \%$ and negative predictive value of $78.2 \%$.Papini et al[10] could predict malignancy in thyroid nodules using hypoechogenicity as criteria with sensitivity of $87.1 \%$, specificity of $43.4 \%$ and positive predictive value of $11.4 \%$. In our study, poorly defined Irregular margins had sensitivity of $44.4 \%$, specificity of $94.12 \%$ and positive predictive value of 57.14\%.Papini et al[10] reported Irregular margins as independent risk factor of malignancy with sensitivity ofn $77.5 \%$,specificity of $85 \%$ and positive predictive value of $30 \%$. Kim et al[9] had sensitivity of $55.51 \%$, specificity of $83 \%$, positive predictive value of $60 \%$ and negative predictive value of $80 \%$. Sajjadieh et al[15] had sensitivity of $42 \%$, specificity of $71 \%$, positive predictive value of $20 \%$, negative predictive value of $87 \%$, when irregular margins were considered. Moifo et al[16] showed sensitivity of $34.78 \%$ and specificity of $99.51 \%$.In present study, microcalcification for predicting malignancy had sensitivity of $33.3 \%$, specificity of $94.12 \%$, positive predictive value of $50 \%$ and negative predictive value of $88.89 \%$.In study conducted by Papini et al[10], sensitivity of microcalcification as a predictor of malignancy was $29 \%$ and Jason et al[13] showed specificity of $94.45 \%$.Moifo et al[16] showed sensitivity of $30.4 \%$ and specificity of $98.8 \%$.In present study, taller than wide for predicting 
malignancy had sensitivity of $22.2 \%$,specificity of $100 \%$,positive predictive value of $100 \%$ and negative predictive value of $87.93 \%$. In study conducted by Moifo et al[16] taller than wide for predicting malignancy had sensitivity of $4.35 \%$,specificity of $100 \%$, positive predictive value of $100 \%$ and negative predictive value of $94.87 \%$.In our study probably benign US findings were seen in 51 patients and probably malignant US findings in 9 patients. Howarth suggested a malignant risk of less than 5\% for TIRADS 3,5\%to10\% for TIRADS4b and greater than $80 \%$ for TIRADS5.Our findings were within this range suggested by Hogarth[8], Russ et al[17] and Moifo et al[16]. Another study conducted by Hong et al[18] concluded that the three sonographic features that are meaningful findings in the diagnosis of thyroid malignancy were the presence of microcacifications,marked hypogenecity and a taller than wide shape. In a multimeter Korean retrospective study, the ultrasound features that were statistically significant for malignancy were hypoechogenicity, marked hypoechogenicity,nonparametric orientation, microlobulated or spiculated margin, an ill defined margins and the presence of microcalcifications.

Table 5:- Summary of statistical performance of major ultrasound features

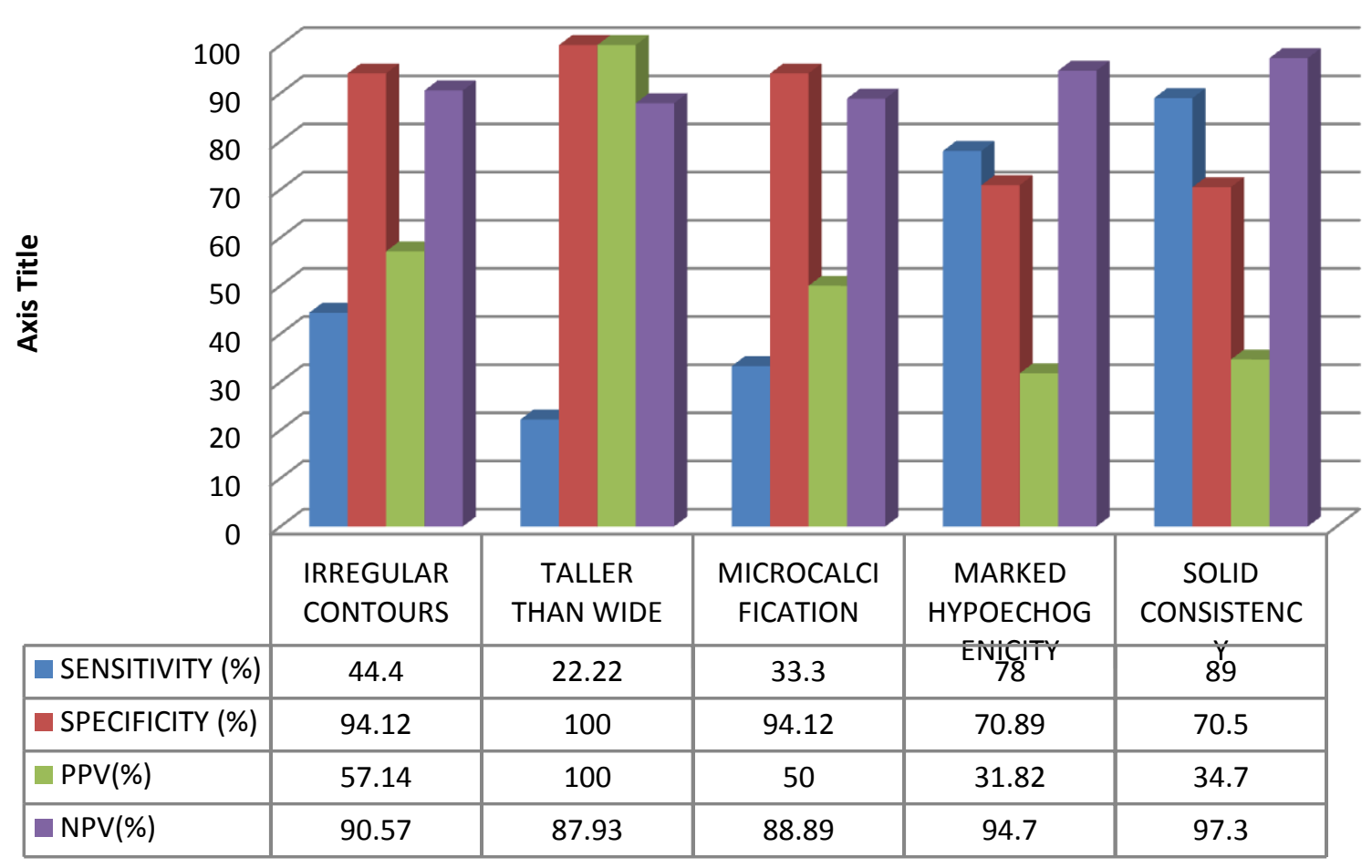

Table 6:- Comparison of risk of malignancy in different TIRADS categories in present study with that of previous published studies

\begin{tabular}{|l|l|l|l|}
\hline TIRADS CATEGORY & Risk Of Malignancy (\%) & Risk Of Malignancy (\%) & Risk Of Malignancy (\%) \\
\hline & Moifo et al (43) & Horvath et al (8) & Present study \\
\hline TIRADS 2 & 0 & 0 & 00 \\
\hline TIRADS 3 & 2.2 & $<5$ & 3.4 \\
\hline TIRADS 4A & 5.9 & $5-10$ & 8.3 \\
\hline TIRADS 4B & 57.9 & $10-80$ & 60 \\
\hline TIRADS 5 & $>80$ & 100 \\
\hline
\end{tabular}

\section{Conclusion:-}

Radiologists should be aware of usefulness of specific ultrasound features of thyroid nodules like Irregular contours, taller than wide configuration, microcalcifications, marked hyoechogenicity and solid consistency collectively taken as TIRADS for better differentiation of benign lesions from malignantor potentially malignant lesions that warrant further diagnostic evaluation. The sensitivity and specificity for Irregular contours were $44.4 \%$ and $94.12 \%$, for taller than wide were $22.22 \%$ and $100 \%$, for microcalcification were $33.3 \%$ and $94.12 \%$, for marked 
hypoechogenicity were $78 \%$ and $70.89 \%$ and for solid consistency were 89 and $70.5 \%$. The risk of malignancy was found to increase from TIRADS 3 to TIRADS5 when the different TIRADS categories were confronted with the results of pathology and risk of malignancy was calculated.

\section{References:-}

1. Mazzaferri EL.Management of a solitary thyroid nodule.N Engl J Med 1993;328;553-59.

2. Aschebrook KB, Ward MH, Sabra MM, DevesaSS.Thyroid cancer incidence patterns in the United States by histological type,1992-2006. Thyroid 2011;21:125-34.

3. Vanderpump MP.The epidemiology of thyroid disease.Br Med Bull 2011;99;39-51.

4. Cooper DS, Doherty GM, Hagen BR, Kloos RT,Lee SLl, Mandela SJet al.American Thyroid Association [ATA] guidelines Taskforce on Thyroid nodules and Differentiated Thyroid Cancer,Revised American Thyroid Association Management guidelines for patients with thyroid nodules and differentiated Thyroid cancer.Thyroid 2009;19:1167-14.

5. Cappelli C,Castellano M, Pirola 1, Cumetti D, Agosti B, Gandossi et al.The predictive value of ultrasound findings in the management of thyroid nodules.Q J Med 2007;100:29-35.

6. Moon WJ, BaekJH, Jungl SL,Kim Dw, Kim Ek, Kim JY et al. Korean Society of Thyroid Radiology[KSTHR]; Korean Society of Radiology.Ultrasonography and the ultrasound based management of thyroid nodules:consensus statement and recommendations.Korean J Radiol 2011;12:1-14

7. kim KM, Park JB, King S,Bae KS. Ultrasonographic guideline for thyroid nodules cytology:single institute experience.J Korean Surg Soc2013;84:73-9.

8. Hovarth E, Majlis S,Rossi R, Franco C, Niedmann JP, Castro A et al.An ultrasonogram reporting system for thyroid nodules stratifying cancer risk for clinical management. J Clinl Endocrinol Metab.2009;94[5]:1748-51.

9. kim Ek,Park CS, Chung WY et al. New sonographic criteria for recommending fine- needle aspiration biopsy of non-palpable solid nodules of thyroid.AJR Am J Roentgenol2002;178[3]:687-91.

10. Papini E,Guglielmi R,Bianchini A et al.Risk of malignancy in nonpalpable nodules; predictive value of ultrasound and colour Doppler Features. J Clin Endocrinol Metab 2002;87[5]:1941-46.

11. Aggarwal Sk,Jayaram G,Kakar A,Goel GD,Parkash R, Pant CS .Fine needle aspiration cytologic diagnosis of solitary cold thyroid nodule.Comparison with Ultrasonography Radionuclide Perfusion study and zero radiography.Acta Cytologica 1989;33[1]:41-47.

12. Koike E, Naguchi S, Yamashita H,Murakmii T.Ultrasonographic characteristics of thyroid nodules;Prediction of malignancy.Arch Surg 2001;136:334-37.

13. Lannuccilli JD,Cronan JJ, Monchik AM.Risk for Malignancy of Thyroid Nodules as Assessed by Sonographic criteria J Ultrasound Med 2004:23:1455-64.

14. Kwak JY, Jung I, Baek JH, Baek SM,Choi N, Choi YJ et al. Korean Society of Thyroid Radiology [KSTHR] and Korean Society of Radiology.Ultrasonography.Imsgr Reporting and characterization System for Ultrasound Features of thyroid Nodules:Multicentric Korean Retrospective Study.Korean J Radiologists.2013 Jan-feb;14[1]:110-17.

15. Sajjadich HR, Sajjadich V, Aminorroaya A, Amini M et al.Value of sonography in determining the nature of Thyroid Nodules: Evaluation of the Sonographic Characteristics. 2005 Jan. Feb. JDMS 21;38-44.

16. Moifo B,Takoeta EO,Tambe J, Blane F, Fortsin JG.Open Journal Of Radiology, 2013,3,103-07.

17. Russ G, Rojer B, Bigorgne C, Rouxel A, Bienvenu M, Laurence P. Prospective Evaluation of Thyroid Imaging And Data System on 4550 Nodules with and without Elastography. European Journal Of Endocrinology [2013] 168:649-55.

18. Hong YJ et al. Positive Predictive Values Of Sonographic Features Of Solid Thyroid Nodules.Clin Imaging March-Apr (2010) 34 [2], 127-33. 